\title{
Excited state structural analysis (ESSA) for correlated states of spin-flip type: application to electronic excitations in nanodiamonds with defects
}

\author{
A.V.Luzanov, O.A.Zhikol \\ STC "Institute for Single Crystals", National Academy of Sciences of \\ Ukraine, 60 Lenin Ave., 61001 Kharkiv, Ukraine
}

Received October 21, 2015

\begin{abstract}
The previously developed ESSA for configuration interaction singles (CIS) method is extended to a more rigorous many-body theory of excited states based on spin-flip (SF) transformations. The so-called SF-CIS (SF approach for CIS) is used, and the respective ESSA indices are constructed. These are atomic excitation indexes $L^{*}$, interatomic chargetransfer numbers $l_{A \rightarrow B}$, and others. By using these quantities, low-lying excitations in the modelled nanodiamonds with color centers (first of all, nitrogen-vacancy (NV) centers) are investigated at a semiempirical level of the theory. It is shown that the lowest excitations are significantly localized in a vicinity of the vacancy. Furthermore, the same excitations are characterized by a high interatomic charge transfer. All these features are common to both types of the $\mathrm{NV}$ centers (neutral $\mathrm{NV}^{0}$ and negative $\mathrm{NV}^{-}$).
\end{abstract}

Keywords: nitrogen-vacancy center, triplet state, interstate excitation, excitation index, charge-transfer number.

Схема ESSA, разработанная ранее для метода однократно возбужденных конфигураций (метод CIS), распространена на более строгую многочастичную теорию возбужденных состояний, которая основывается на преобразованиях спин-флип (SF). Используется так называемая модель SF-CIS (SF в отношениии CIS), для которой сконструированы соответствующие индексы ESSA. Это - атомные индексы возбуждения $L^{*}$, межатомные числа переноса заряда $l_{A \rightarrow B}$ и другие. C помощью этих величин исследованы нижайшие возбуждения в модельных наноалмазах с центрами окраски (прежде всего, азот-вакансионные (NV) центры). Найдено, что данные возбуждения в значительной степени локализованы в непосредственной близости от вакансии и характеризуются высоким уровнем переноса заряда. Все указанные черты присущи обоим NV-центрам - нейтральному $\mathrm{NV}^{0}$ и отрицательному $\mathrm{NV}^{-}$.

Структурний аналіз збудженних станів (ESSA) для корельованих станів за типом спін-фліп: застосування до електронних збуджень у наноалмазах із дефектами. А.В.Лузанов, О.А.Жикол.

Схему ESSA, що її раніше розвинуто стосовно методу однократно збуджених конфігурацій (метод CIS), поширено на більш строгу багаточастинкову теорію збуджених станів, яка грунтується на спін-фліп (SF) перетвореннях. Використовано так звану модель SF-CIS (SF щодо CIS), для якої сконструйовано відповідні індекси ESSA. Це - атомні індекси збудження $\mathrm{L}_{A}$, міжатомні числа переносу заряда $l_{A \rightarrow B}$ та інші. Із застосуванням цих величин досліджено найнижчі збудження модельних наноалмазів із центрами забарвлення, в першу чергу, нітроген-вакансійні (NV) центри. Знайдено, що ці збудження значною мірою локалізовані у безпосередній близькості від вакансії і характеризуються високим рівнем зарядового переносу. Всі означені риси притаманні як нейтральному $\mathrm{NV}^{0}$, так i негативному $\mathrm{NV}^{-}$центрам. 


\section{Introduction}

Study of electronic properties of molecular excited states is one of the mainstreams in the molecular quantum mechanics and its applications. Now it is even more topical, because the excited-state spectroscopy became an efficient tool for the experimental study of optically excited states of chemical species. The latter are very important for examining molecular aggregats, huge atomic clusters, nanocrystals with defects, etc. $[1,2]$. New possible applications in nanoscience and future quantum information processing (spin-logic devices) are in part responsible for the contemporary resurgence of interest in high-spin vacancies and other local defect states occurring in nanoclusters, particularly in nanodiamonds and silicon carbides [3,4]. All this motivates to extend or improve existing quantum chemistry techniques for excited molecules with stress on the theoretical description and interpretation of excited states in large-scale systems.

Today, time-dependent density functional theory (TDDFT) [5] has become the main workhorse for obtaining approximate theoretical spectra in spectrochemistry and material science. Although TDDFT fails in certain cases (see [6] and references therein), it remains to be the basic model for complex polyatomic systems. It is also worth mentioning that the precursor of TDDFT was the so-called random phase approximation (RPA) earlier elaborated in many-body theory [7-9]. In turn, RPA is closely related to the Tamm-Dancoff approximation that appeared in quantum chemistry in another disguise. It is the conventional scheme of configuration interaction singles (CIS). For describing excited states, CIS is a quite successful model at the semiempirical level. At the same time, poor performance of the $a b$ initio CIS is common. That is why density functional (DFT) methods, such as TDDFT and CIS-DFT [10] (i.e., a DFT counterpart of CIS), have proved to be a fundamental tool within excited-state calculation machinery and its applications to materials science. We must also mention another important analog of CIS. This is the spin-flip CIS and spin-flip DFT techniques given and further developed in [11-14]. We see that many significant models are based on CIS and CISlike correlated wave-function constructions. This fact is the starting point of our present study of excited states.

Before proceeding further, a few words should be said about the methods for analyzing excited states. For CIS excited states, the consistent interpretation based on transition density matrix was presented long ago [15-17]. In our subsequent work [18] this approach, named as excited state structural analysis (ESSA), was extended to TDDFT. Various generalizations of ESSA (or schemes mimicking ESSA) can be found in several recent works [19-24]. However, they usually have a somewhat restrictive range of applicability for highly correlated states (when contributions from double excitations are significant). It seems to us that it is practically impossible to formulate a full and consistent ESSA scheme for arbitrary excited state wave functions. Apparently, one must develop ad hoc special ESSA tools for specific models, as done in the recent studies for EOM-CCSD excited states [25] and for weakly coupled chromophores [26].

The aforementioned spin-flip CIS (SFCIS) approximation is likely to be one of simplest and often effective models for excited states in systems having a quasi-degenerate ground state. It is just such highly correlated states occurring in typical point defects, which are our principal concern in this paper. Thus, the main purpose of the paper is to develop a consistent ESSA for the SF-CIS and related spin-flip DFT methods. The proposed ESSA technique will be applied in detail to elucidate a nature of the excited states in some color centers of nanodiamonds.

\section{ESSA for usual CIS-like excitations}

We start with outlining ESSA [15-17] for the usual CIS states. This ESSA approach will serve below as a suitable pattern for the modified formulation applicable to interstate electron transitions as well. Stress that the original ESSA is applicable for excitations from the ground state only. The attractive advantage of ESSA for CIS is that excitations from the ground state can be consistently described by the charge transfer (CT) numbers first given in [16] (see also reviews $[17,19]$ ). From these CT numbers the other indexes can be naturally produced within the CIS theory. Here we briefly describe this ESSA pattern.

Let us consider a molecule in the given excited state, and let us define CT number $l_{A \rightarrow B}$ as a full probability of electron transfer (under excitation) from atom $A$ to atom $B$. By definition, number $l_{A} \equiv l_{A \rightarrow A}$ is a partial localization index for atom $A$. The full excitation localization index $L^{*}{ }_{A}$ (excitation index) is defined as a probability that atom $A$ participates in all excitation "sub- 
processes", that is in CT and local excitations. Explicitly it means that

$$
L_{A}^{*}=\sum_{B}\left(l_{A \rightarrow B}+l_{B \rightarrow A}\right) / 2,
$$

or

$$
L_{A}^{*}=l_{A}+\sum_{B \neq A}\left(l_{A \rightarrow B}+l_{B \rightarrow A}\right) / 2 .
$$

The set $\left\{L_{A}^{*}\right\}$ is naturally normalized to unity.

The introduced CT numbers will be physically consistent measures of CT if they correctly reproduce a redistribution of atomic electron charges due to excitation. Let us denote by $\Delta q_{A}{ }^{*}$ an excitation-produced variation of the total charge on atom $A$ (with a sign convention). Then one might expect also that a balance relation

$$
\Delta q^{*}{ }_{A}=\sum_{B}\left(l_{A} \rightarrow B-l_{B \rightarrow A}\right)
$$

should be valid. The ESSA scheme for CIS in [16, 17] completely agrees with Eqs. (1) and (2).

More specifically, in case of the CIS model we have the excited-state wave function of the form

$$
|\Psi C I S\rangle=\sum_{1 \leq i \leq N} \tau(i)|\Phi\rangle,
$$

where $|\Phi\rangle$ is a reference Slater determinant (in fact, the ground state wave function). In Eq. (3) elementary transition matrix $\tau$ produces substitutions of occupied (in $|\Phi\rangle$ ) spin-orbitals by vacant spin-orbitals. This matrix should be normalized to unity $\left(\operatorname{Tr} \tau^{+} \tau=1\right)$. In that case the $\tau$ matrix elements can be used as probability amplitudes of appropriate transition processes. For instance, let $\left\{\tau_{\mu \nu}\right\}$ be a set of such amplitudes computed from $\tau$ in a basis of orthonormal atomic spin-orbitals. Then, the key indexes, that is CT numbers, should be evaluated in accordance with [16-8] as follows:

$$
l_{A \rightarrow B}=\sum_{\mu \in A} \sum_{\nu \in B}\left|\tau_{\nu \mu}\right|^{2} .
$$

For these numbers, we will make use of a more detailed notation $l_{A \rightarrow B}[j]$, if the given CIS excited state needs to be specified explicitly by $\left|\Psi_{j}^{C I S}\right\rangle$ and the corresponding matrix $\tau_{j}$. The above outlined ESSA pattern which satisfies Eqs. (1) and (2), is termed here a canonical ESSA.

\section{Extension to interstate transitions}

Now we extend the canonical ESSA procedure to the broader problem of finding excitation indexes and CT numbers for transitions between excited electronic states. For this we turn to our initial work [15] concerning ESSA for usual excitations. In it we suggested that nonnegative functions (algebraic invariants) of density matrix difference rather than moduli of charge density or bond order differences should be taken as underlying entities for making ESSA.

Let us define an additional notion: $\Delta^{i \rightarrow j}$ is the one-electron density matrix difference for transition between the $i$ th and $j$ th excited states, or

$$
\Delta^{i \rightarrow j}=D_{j}^{*}-D_{i}^{*},
$$

where $D_{i}{ }^{*}$ denotes the density matrix for $i$ th excited state. For making ESSA we have to form the (normalized) matrix modulus of $\Delta^{i \rightarrow j}$. This is easy to be done by reducing $\Delta^{i \rightarrow j}$ to a diagonal form, and replacing in it all diagonal elements with their absolute values. Converting the thus obtained diagonal matrix to the initial basis representation, one gets the required matrix modulus $\left|\Delta^{i \rightarrow j}\right|$. Then, following $[15,18]$, the excitation localization operator should be of the form

$$
\hat{L^{i \rightarrow j}}=\left(\operatorname{Tr}\left|\Delta^{i \rightarrow j}\right|\right)^{-1}\left|\Delta^{i \rightarrow j}\right| .
$$

From this the respective excitation indexes can be defined in the usual manner by using the corresponding diagonal matrix elements:

$$
L_{A}^{i \rightarrow j}=\sum_{\mu \in A}\left(\hat{L^{i \rightarrow j}}\right) \mu \mu \cdot
$$

In fact, this expression was previously considered in [18]. Here we go further, and for these interstate excitations the appropriate CT numbers will be proposed. To this aim, we write down the explicit expression for the CIS matrix $D_{j}{ }^{*}$ in terms of the associated transition matrix $\tau_{j}$, namely [18]

$$
D_{j}^{*}=\rho+\tau_{j} \tau_{j}^{+}-\tau_{j}^{+} \tau_{j},
$$

with $\rho$ being the conventional density matrix of the reference Slater determinant. Identifying $\Delta q_{A}{ }^{*}$ with expression of type (7) where we have to replace $\hat{L}^{i \rightarrow j}$ with $\Delta^{i \rightarrow j}$ from Eq. (5), and doing some algebra we get 


$$
\Delta q_{A}^{*}=\sum_{B}\left(l_{A}^{[j]} \rightarrow B+l_{B \rightarrow A}^{[i]}-l_{B \rightarrow A}^{[j]}-l_{A}^{[j]} \rightarrow B\right) .
$$

Thus, the idea comes to mind to identify the first two terms under sum sign in Eq. (9), with the sought CT numbers. More exactly, in the case of interstate excitations the quantities

$$
l_{A \rightarrow B}^{i \rightarrow j}=\left(l_{A}^{[j]} \rightarrow B+l_{B \rightarrow A}^{[i]}\right) / 2
$$

will serve us as counterparts of CT numbers (2). The factor $1 / 2$ is introduced in order for the unit normalization condition $\sum_{A \rightarrow B} l_{A \rightarrow B}^{i \rightarrow j}=1$ to be satisfied. In so doing, the counterpart of the balance equation (2) is now of the form

$$
\Delta q_{A}^{*}=2 \sum_{B}\left(l_{A \rightarrow B}^{i \rightarrow j}-l_{B \rightarrow}^{i \rightarrow j}\right) .
$$

Thus, we obtained the full set of equations determining ESSA for interstate CIStype excitations. In principle, the same expression (10) can be applied beyond the CIS approximation. One point should be stressed here. The excitation indexes, evaluated from Eq. (7), do not satisfy Eq. (1) of the canonical ESSA, that is excitation indexes $L_{A} i \rightarrow j$ and CT indexes $l_{A \rightarrow B}^{i \rightarrow j}$ are now independent quantities, unlike their canonical analogues. Apparently, it is connected with a more complex electron-configuration structure of interstate transitions even within the CIS approximation of the individual excitations.

\section{Using correlated excited states of spin-flip type}

We focus now on SF-CIS model as a special version of the CIS theory. Explicitly, this model was introduced in [11] at ab initio level, although there as well existed earlier semiempirical formulation [27] of a somewhat similar spin-flip methodology. As mentioned in the introduction, the fundamental property of SF-CIS and related approaches is their ability to treat quasi-degenerate electronic states. In addition, it is important that SF-CIS is able to be efficiently reformulated in a manner that allows the model to be incorporated into the TDDFT formalism [12, 13]. These facts prompted us to elaborate ESSA for excited states produced by SF-CIS techniques. The generalization of ESSA in the preceding section just suits our purpose.
We investigated SF-CIS previously [28] and additionally in [29], and here we will avoid going into detail as to the SF-CIS computational technique. In the present paper context, only few formal matters should be mentioned. They are related to a choice of reference determinant $|\Phi\rangle$ and transition matrix $\tau$ in Eq. (3). Let the excited state of interest have total spin value $S_{\text {target }}$. Then, we first precompute the ROHF (restricted open-shell Hartree-Fock) wave function corresponding to the higher total spin $S_{0}=S_{\text {target }}+1$. Starting with this reference we compute the variationally optimized SF-CIS state vector $|\Psi S F-C I S\rangle$ of the form (3) with a transition matrix of the special form:

$$
\tau=t|\beta\rangle\langle\alpha|
$$

with $t$ being a spinless matrix of coupling amplitudes, and $|\alpha\rangle$ and $|\beta\rangle$ being the usual spin-up and spin-down eigenstates, respectively. Evidently, the spin dyad $|\beta\rangle\langle\alpha \|$ in Eq. (12) makes a spin-flip transformation, thus generating the required low-spin electronic configuration. As to spin contamination problems in SF models, see [28]. Usually, the SF-CIS lowest-energy states have a small spin contamination, so $S_{\text {target }}$ is a good quantum number.

To obtain low-lying excitations we have to consider the minimal energy SF-CIS state, $\left|\Psi_{1}^{S F-C I S}\right\rangle$, and a higher-energy state $|\Psi \underset{j}{S} F-C I S\rangle, j \geq 2$. Each of these states is described by its own coupling amplitude matrix $t$, which we will denote as $t_{1}$ and $t_{j}$, respectively. Thus, bearing in mind Eq. (12), we can directly apply relations of the preceding section, and produce new indexes $l_{A \rightarrow B}^{(j)}$ [SF-CIS]. They surve us as a counterpart of CT numbers corresponding to the $j$ th SF-CIS excitation from the ground SF-CIS state. By using notation

$$
l_{A \rightarrow B}[t]=\sum_{\mu \in A} \sum_{v \in B}\left|t_{v \mu}\right|^{2},
$$

we have the following working formula for CT number

$$
l_{A \rightarrow B}^{(j)}[\mathrm{SF}-\mathrm{CIS}]=\left(l_{A \rightarrow B}\left[t_{j}\right]+l_{B \rightarrow A}\left[t_{1}\right]\right) / 2(1
$$

Excitation indexes $L_{A}{ }^{*} \equiv L_{A}{ }^{i \rightarrow j}$ [SF-CIS] need a bit more computations. To evaluate them we must, in accordance with Eq. (8), take into account structure (12) of transi- 
tion matrices $\tau_{1}$ and $\tau_{j}$. The details are given in Appendix. As a result, we have the specialized ESSA scheme for the excited states allowing for electron correlation effects beyond the conventional CIS model.

Before proceeding with the main demonstrations, it is advisable to compare the results of SF-CIS and full CI (FCI) approaches. The last provides exact solutions within the matrix approximation of the total molecular Hamiltonian by the finitedimensional atomic-orbital basis. For FCI excitations we apply the same approach based on Eqs. (6) and (7). We will treat in a non-empirical way a small linear hydrogen cluster $\mathrm{H}_{10}$ with the nearest interatomic distances as in the normal $\mathrm{H}_{2}$ molecule. Using the minimal atomic-orbital basis set of STO$4 \mathrm{G}$ quality, we calculated the first electronic transition $1 \sum_{g} \rightarrow 1 \sum_{u}$. For this transition we obtained the following distributions of the excitation over the atoms in $\mathrm{H}_{10}$ chain (the values for nonequivalent sites starting from the end atom):

$L_{A}^{*}[\mathrm{FCI}]=0.208,0.021,0.139,0.045,0.087$,

$L_{A}^{*}[\mathrm{~S} F-\mathrm{CIS}]=0.215,0.011,0.151,0.036,0.086$

Another example is the lowest singlet excitation in biradical molecule HNO:

$$
\begin{gathered}
L_{A}^{*}[\mathrm{FC}]=0.049,0.306,0.645, \\
L_{A}^{*}[\mathrm{SF}-\mathrm{CIS}]=0.025,0.302,0.673 .
\end{gathered}
$$

Both examples demonstrate a quite satisfactory performance of ESSA for SF-CIS.

Futhermore, we also need to validate the same SF-CIS technique at a semiempirical level. The usual CNDO (complete neglect of differential overlap) scheme remains a quite useful approach to huge molecular clusters, and we test the method on the example of carbene $\mathrm{C}=\mathrm{CH}_{2}$ (vinylidene). The results are given in Table 1 where along with the excitation distribution $\left\{L_{A}{ }^{(j)}\right.$ for $j=1,2,3$ we present the corresponding excitation energies $\lambda_{0 \rightarrow j}$, and oscillator strengths $f_{0 \rightarrow j}$. Other systems also show satisfactory results, so that the suggested spin-flip approach ESSA/SFCIS can be accepted as a reasonable tool for analyzing low-lying excitations.
Table 1. Comparison of FCI and SF-CIS results for the lowest three excited states of the vinylidene molecule treated at the CNDO

\begin{tabular}{|c|c|c|}
\hline$j$ & $\lambda_{0 \rightarrow j}\left(f_{0 \rightarrow j}\right)$ & $\left\{L_{A}^{(j)}\right\}$ \\
\hline 1 & $\frac{3.86}{3.85} \frac{(0.005)}{(0.010)}$ & $\frac{\{0.110}{\{0.081}, \frac{0.750}{0.779}, \frac{0.070\}}{0.060\}}$ \\
\hline 2 & $\frac{5.00}{4.82}(\underline{0 .})$ & $\begin{array}{l}\{0.259 \\
\{0.282\end{array}, \frac{0.605}{0.630}, \frac{0.068\}}{0.044\}}$ \\
\hline 3 & $\frac{6.90}{6.72}(0.160)$ & $\left\{0.312, \frac{0.646}{\{0.301}, \frac{0.021\}}{0.663}, \frac{0.017\}}{0.0}\right.$ \\
\hline
\end{tabular}
level. The FCI data are underlined.

\section{Applications to defects in nanodiamonds}

Here, excited states of nanodiamonds with point defects are analyzed by ESSA/SF-CIS. As mentioned in the introduction, nanodiamonds are very important materials with broad spectrum of properties and applications [30,31]. Especially thorough are the investigations of nitrogen-vacancy color centers (NV-centers) in various diamond structures (see reviews [3, 32-34]). At present, NV-centers are considered as a promising candidate for qubit (quantum bit) in quantum information processing [35-37]. It may be observed that the recent book [38] provides a clear and very pedagogical introduction to the quantum computation theory.

As in our previous study [29], we consider a finite-size carbon cluster with the idealized geometry as in the diamond lattice. Specifically, we take the one-hundred atom cluster terminated by hydrogen atoms. The built molecular structure $\mathrm{C}_{100} \mathrm{H}_{72}$ has the $D_{3 d}$ symmetry group. The atomic structure of NV-centers is generated from $\mathrm{C}_{100} \mathrm{H}_{72}$ by replacing one inner carbon atom by the nitrogen atom, and, additionally, by making a vacancy at the nearest-neighbor site (see the image in [29], Table 3). The most important is the case of charged $\left(\mathrm{NV}^{-}\right)$defect, and studying this we will deal with cluster $\mathrm{C}_{98} \mathrm{NH}_{72}{ }^{-}$. We performed SF-CIS/CNDO computations on this structural defect and obtained the respective energies of the ground and lowest excited states. In the used model, the ground state of $\mathrm{NV}^{-}$center is of triplet nature in agreement with the main experimental and theoretical data [32]. The ground state will be denoted as $T_{0}$ and the first triplet excited states as $T_{1}$ and $T_{2}$. For these $T-T$ transitions we display diagrams of the atomic excitation distribution $\left\{\mathrm{L}_{A}{ }^{*}\right\}$ 
Table 2. Excitation localization distribution $\left\{L_{A}^{*}\right\}$ (red color) for the two lowest $T_{0} \rightarrow T_{j}$ transitions and $D_{0} \rightarrow D_{1}$ transition in the modelled $\mathrm{NV}^{-}$and $\mathrm{NV}^{0}$-centers, respectively, within SF-CIS/CNDO.

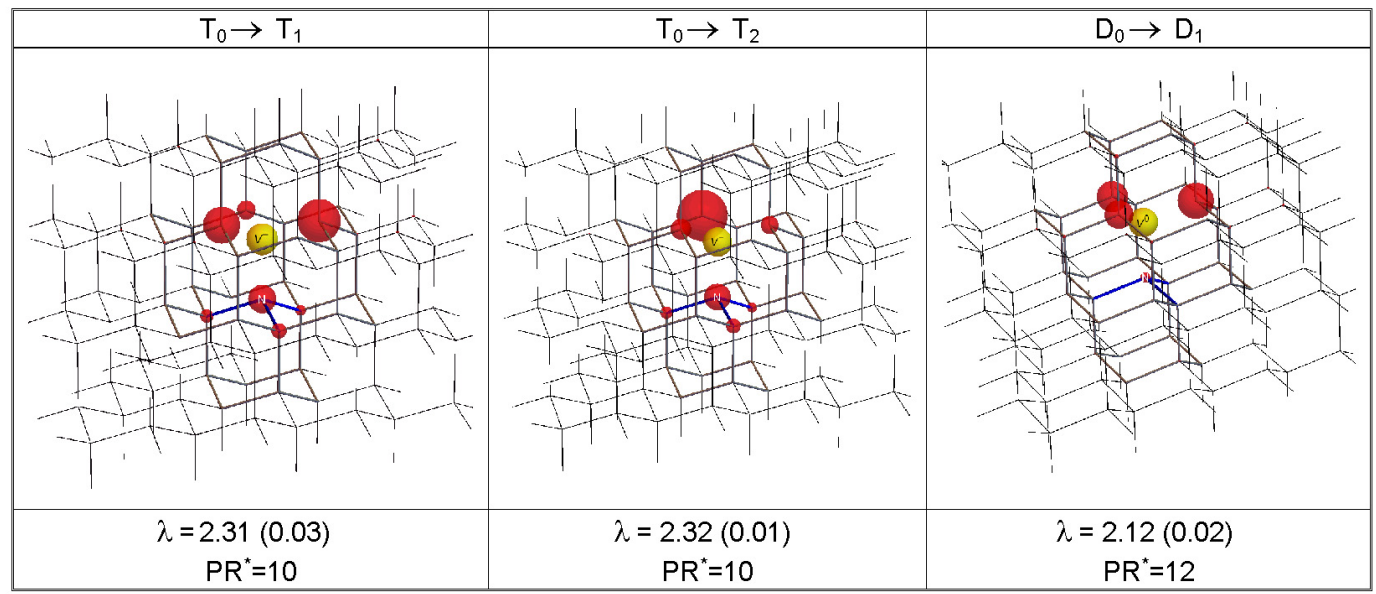

Table 3. Difference charge densities distribution $\left\{\Delta q_{A}^{(j)}\right\}$ for the two lowest $T_{0} \rightarrow T_{j}$ transitions and $D_{0} \rightarrow D_{1}$ transition in the modelled $\mathrm{NV}^{-}$and $\mathrm{NV}^{0}$-centers, respectively, within SF-CIS/CNDO (violet color for positive charge differences, cyan color for negative ones). In the last row, exciton radius $R_{\text {exc }}$ (in $\AA$ ) and charge transfer index $\mathrm{CT}_{\text {tot }}$ (in \%) are given.

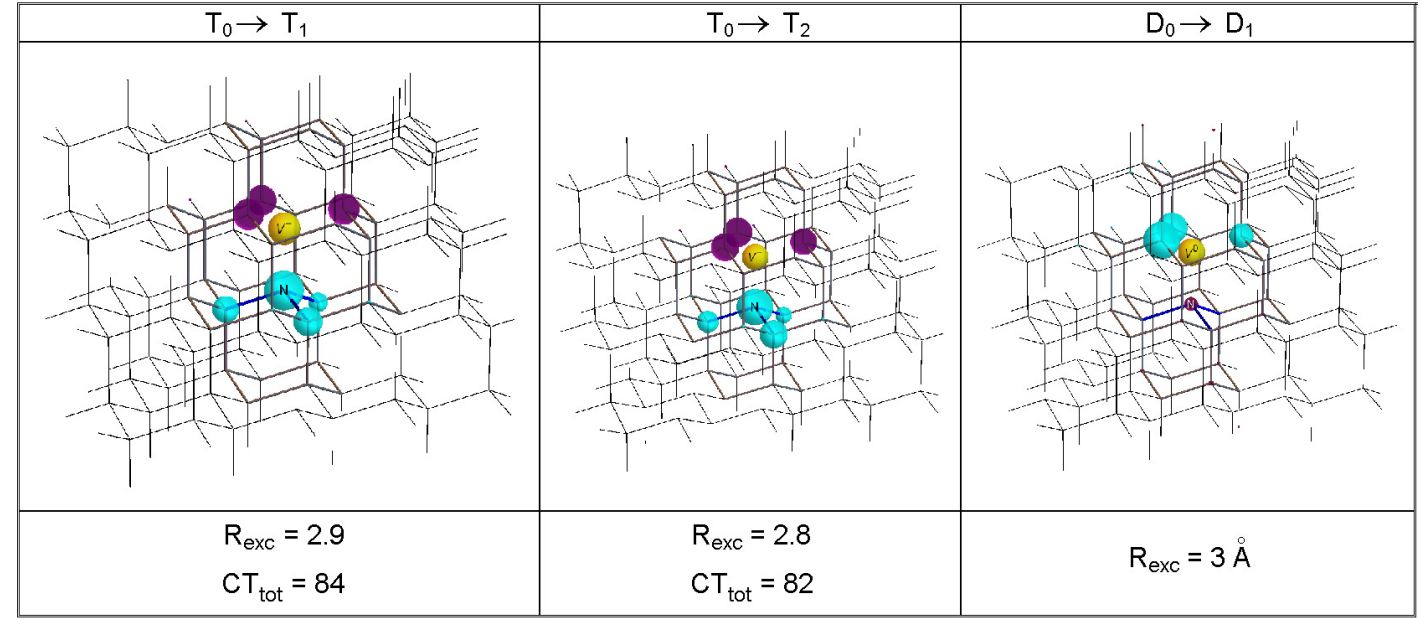

(Table 2) and difference charge density distribution $\left(\Delta q_{A}{ }^{*}\right)$ (Table 3$)$.

In Table 3, additional ESSA indexes are given, namely, excitation radius, $R_{\text {exc }}$, and average CT index, $\mathrm{CT}_{\text {tot }}$. They are defined in [18] by Eq. (6.28), and by Eq. (14.50) in [19], respectively. According to [19], index $\mathrm{CT}_{\text {tot }}$ is computed as follows:

$$
\mathrm{CT}_{\text {tot }}=1-\sum_{A} l_{A \rightarrow A}
$$

or

$$
\mathrm{CT}_{\text {tot }}=\sum_{A \neq B} l_{A \rightarrow B}
$$

It gives an average measure of interatomic CT processes. Furthermore, in Table 2 a relevant $\mathrm{PR}^{*}$ (partition ratio) index for $\left\{L_{A}{ }^{*}\right)$ is added. We define $\mathrm{PR}^{*}$ as follows:

$$
\mathrm{PR}^{*}=1 / \sum_{B}\left(L_{A}^{*}\right)^{2}
$$

Notice that inverse partition ratio is the well-known measure of average localization related to the given probability distribution $[39,40]$. In our case it estimates a mean number of the atoms involved in excitation.

From Table 2 we see that the lowest triplet-triplet excitations ( $T-T$ transitions) are localized in the nearest vicinity of a vacancy site adjacent to a substitutional nitro-

Functional materials, 23, 1, 2016 
gen atom. Unlike the spin density distribution in the ground state $T_{0}$ (see Table 3 in [29]), the nitrogen atom markedly participates in the excitation. The $\mathrm{PR}^{*}=10$ is consistent with high degree of localization of the studied $T-T$ excitations. Notice that these excitations are quasi-degenerate, as it should be for the used finite-size model of $\mathrm{NV}^{-}$defect [29].

The analysis of the difference charge density (see images in Table 2) comes to the same conclusion about the basically local character of the $T-T$ transitions. At the same time, a large CT index $\mathrm{CT}_{\text {tot }}$ and $\mathrm{R}_{\text {exc }}$ values (about twice length of $\mathrm{C}-\mathrm{C}$ bond) tell us that these local transitions have a significant charge-transfer structure. This structure is generated by electron transfers between nonadjacent atoms of the first coordination sphere of the vacancy site. Three carbon atoms adjacent to the vacancy lose in total 0.38 and 0.42 electron for $T_{0} \rightarrow T_{1}$ and $T_{0} \rightarrow T_{2}$ transitions, respectively. In case of the neutral color center $\mathrm{NV}^{0}$ we analyzed the averaged characteristics of the lowest quasi-degenerate doublet excitation $D_{0} \rightarrow D_{1}$ for which we observed the same features (localization near vacancy etc.) as in the charged $\mathrm{NV}^{-}$center. For instance, the three above-mentioned atoms gain 0.45 electron; however, the direction of charge transfer is inverted.

\section{Conclusions}

In this paper we proposed the ESSA-type scheme which allows to analyze the electronic structure of complex excited states in visual terms. For the investigated spin-flip model we defined the appropriate one-center excitation index $L_{A}{ }^{*}$ as a total probability for atom $A$ to be involved into the given electronic transition. The two-center chargetransfer index $l_{A \rightarrow B}$ quantifies a probability of excited electron to pass from atom $A$ to atom $B$. Taken together, these indexes, along with other characteristics (excitation radius, average CT measure, etc.) provide a detailed interpretation of excitations in molecules and atomic clusters. Here we applied the approach to the modelled nanodiamond clusters with NV color centers. The computations revealed some interesting details concerning the nature of low-lying excitations in these systems (a rather strong localization in vicinity of defects, a high CT character etc.). On the whole, the proposed semiempirical model describes satisfactorily the considered NV defects. Notice that programming and computations were undertaken within the Wolfram Mathematica environment [41]. By using a more powerful software we may essentially extend the practical efficiency of the approach. It is also interesting to apply the developed approach to similar nanodiamond clusters treated by the spin-flip approximation within the current DFT machinery.

In context of quantum information processing, a set of various spin-logic devices is desirable to be designed, and study of functionalized nanodiamonds and nanodiamonds with subsurface-localized color centers provide another interesting avenue for future work. As an example we point to the problem of neutral OV-center, that is isoelectronic oxygen cousin of $\mathrm{NV}^{-}$center. This system was first studied in [42] where it is shown that OV center has the triplet ground state like $\mathrm{NV}^{-}$center. More full quantum chemistry computations of $\mathrm{OV}$ center, including its optical properties, was made quite recently [43, 44]. Functionalized systems of this type are also perspective objects to be explored in the framework of the ESSA technique.

Acknowledgement. The authors are thankful to A.P.Nizovtzev and V.A.Pushkarchuk for useful comments and discussion. This research was supported by the joint project of the National Academy of Sciences of Ukraine and the National Academy of Sciences of Belarus (Grant No.09-06-15).

\section{Appendix. Excitation inidexes for the spin-flip model}

Here we describe how to compute atomic excitation indexes $L_{A}{ }^{*} \equiv L_{A}{ }^{1 \rightarrow j}$ [SF-CIS]. For this we must specify matrix modulus $\left|\Delta^{1 \rightarrow j}\right|$ of the difference density matrix

$$
\Delta^{1 \rightarrow j}=D_{j}^{*}-D_{1}^{*} .
$$

In the present context, $D_{j}{ }^{*}$ and $D_{1}{ }^{*}$ are defined as in Eq. (8) but with matrices $\tau_{j}$ of the form (12). It gives the intermediate expression

$$
\Delta^{1 \rightarrow j}=\Delta_{\alpha}^{1 \rightarrow j}|\alpha\rangle\left\langle\alpha\left|+\Delta_{\beta}^{1 \rightarrow j}\right| \beta\right\rangle\langle\beta|,
$$

where $t$

$$
\Delta_{\alpha}^{1 \rightarrow j}=-\left(t_{j}^{+} t_{j}-t_{1}^{+} t_{1}\right), \quad \Delta_{\beta}^{1 \rightarrow j}=t_{j} t_{j}^{+}-t_{1} t_{1}^{+} .
$$

We stress that due to $\langle\alpha \mid \beta\rangle=0$ matrices $\Delta_{\alpha}{ }^{1 \rightarrow j}$ and $\Delta_{\beta}{ }^{1 \rightarrow j}$ are working in the different orthogonal spin-orbital subspaces, so 


$$
\left|\Delta^{1 \rightarrow j}\right|=\left(\left|\Delta_{\alpha}^{1 \rightarrow j}\right|\right)|\alpha\rangle\left\langle\alpha\left|+\left(\left|\Delta_{\beta}^{1 \rightarrow j}\right|\right)\right| \beta\right\rangle\langle\beta| .
$$

Using Eq. (A3), we easily compute matrix molduli $\left|\Delta_{\alpha}{ }^{1 \rightarrow j}\right|$ and $\left|\Delta_{\beta}{ }^{1->j}\right|$ (see the text in section 3$)$.

From Eq. (A4), it follows that the normalization factor in Eq. (6) is

$$
N_{\alpha \beta} \equiv \operatorname{Tr}\left|\Delta^{1 \rightarrow j}\right|=\operatorname{Tr}\left|\Delta_{\alpha}^{1 \rightarrow j}\right|+\operatorname{Tr}\left|\Delta_{\beta}^{1 \rightarrow j}\right|,
$$

and the needed excitation indexes are of the form

$$
L_{A}^{*}=\sum_{\mu \in A}\left[\left|\Delta_{\alpha}^{1 \rightarrow j}\right|_{\mu \mu}+\left|\Delta_{\beta}^{1 \rightarrow j}\right|_{\mu \mu}\right] / N_{\alpha \beta} \cdot
$$

By Eqs. (A3), (A5) and (A6) the computations of the SF-CIS excitation indexes are fully determined.

\section{References}

1. G.D.Fuchs, V.V.Dobrovitski, R.Hanson et al., Phys. Rev. Lett., 101, 117601 (2008).

2. P.Neumann, R.Kolesov, V.Jacques et al., New J.Phys., 11, 013017 (2009).

3. V.V.Dobrovitski, G.D.Fuchs, A.L.Falk et al., Annu.Rev.Condens. Matter Phys., 4, 23 (2013).

4. A.Morero, Nat. Mater., 14, 135 (2015).

5. Fundamentals of Time-dependent Densityfunctional Theory, ed. by M.A.L.Marques, N.Maitra, F.Nogueir et al., Lecture Notes in Physics, Springer, Berlin (2012).

6. P.Elliott, S.Goldson, C.Canahui, N.T.Maitra, Chem. Phys., 391, 110 (2011).

7. N.N.Bogolubov, Lectures on Quantum Statistics, Gordon \& Breach, New York (1970).

8. D.J.Rowe, Rev.Mod.Phys., 40, 153 (1968).

9. J.P.Blaisot, G.Ripka, Quantum Theory of Finite Systems, Massachusetts Institute of Technology, Massachusetts (1986).

10. S.Hirata, M.Head-Gordon, Chem.Phys.Lett., 314, 291 (1999)

11. A.I.Krylov, Chem.Phys.Lett., 338, 375 (2001).

12. Y.Shao, M.Head-Gordon, A.I.Krylov, J.Chem. Phys., 118, 4807 (2003).

13. Y.A.Bernard, Y.Shao, A.I.Krylov, J.Chem. Phys., 136, 204103 (2012).

14. X.Zhang, J.M.Herbert, J.Chem.Phys., 141, 064104 (2014).

15. A.V.Luzanov, A.A.Sukhorukov, V.E.Umanskii, Teor. Eksper. Khim., 10, 456 (1974).

16. A.V.Luzanov, V.F.Pedash, Theor.Exp.Chem., 15, 338 (1979).

17. A.V.Luzanov, Russ.Chem.Rev., 49, 1033 (1980).

18. A.V.Luzanov, O.A.Zhikol, Int.J.Quantum Chem., 110, 902 (2010).

19. A.V.Luzanov, O.A.Zhikol, in: Practical Aspects of Computational Chemistry I: An Over- view of the Last Two Decades and Current Trends, ed. by J.Leszczynski, M.K.Shukla, Springer, New York (2012), p.415.

20. F.Plasser, H.Lischka, J.Chem. Theory Comp., 8, 2777 (2012).

21. F.Plasser, S.A.Bappler, M.Wormit, A.Dreuw, J.Chem.Phys., 141, 024107 (2014); S.A.Bappler, F.Plasser, M.Wormit, A.Dreuw, Phys. Rev.A，90, $052521 \quad$ (2014); F.Plasser, B.Thomitzni, S.A.Bappler et al., J.Comput. Chem., 36, 1609 (2015).

22. L.Blancafort, A.A.Voityuk, J.Chem.Phys., 140, 095102 (2014).

23. T.Etienne, X.Assfeld, A.Monari, J.Chem.Theory Comput., 10, 3896 (2014).

24. T.Etienne, J.Chem. Theory Comput., 11, 1692 (2014); J.Chem. Phys., 142, 244103 (2015).

25. A.V.Luzanov, Functional Materials, 21, 125 (2014).

26. A.V.Luzanov, D.Casanova, X.Feng, A.I.Krylov, J.Chem. Phys., 142, 224104 (2015).

27. A.V.Luzanov, Theor.Exp.Chem., 17, 227 (1982); Ibid, 27, 356 (1991).

28. A.V.Luzanov, J. Struct. Chem., 44, 729 (2004).

29. A.V.Luzanov, Functional Materials, 22, 514 (2015).

30. Physics and Applications of CVD Diamond, ed. by C.Nebel, M.Nesladek, Wiley, Weinheim (2008).

31. Optical Engineering of Diamond, ed. by C.R.P.Mildren, J.R.Rabeau, Wiley, Berlin (2013).

32. M.W.Doherty. N.B.Manson, P.Delaney et al., Phys. Reports, 528, 1 (2013).

33. R.Schirhagl, K.Chang, M.Loretz, C.L.Degen, Annu.Rev.Phys.Chem., 65, 83 (2014).

34. Quantum Information Processing with Diamond, ed. by S.Prawer, I.Aharonovich, Elsevier, Cambridge, UK (2014).

35. J.Wrachtrup, S.Y.Kilin, A.P.Nizovtsev, Opt. Spectrosc., 91, 429 (2001).

36. J.J.L.Morton, J. Elzerman, Nature Nanotechnology, 9, 167 (2014).

37. H.Bernien, B.Hensen, W.Pfaff, Nature, 497, 86 (2013).

38. V.V.Yanovski, Quantum Mechanics of Algorithms, ISMA, Kharkiv (2009) [in Russian].

39. R.J.Bell, P.Dean, D.C.Hibbins-Butler, J.Phys. $C, 3,2111(1970)$

40. N.C.Murphy, R.Wortis, W.A.Atkinson, Phys. Rev. B, 83, 184206 (2011).

41. Wolfram Research, Inc., Mathematica, Version 5.2, Champaign, IL (2005).

42. J.P.Goss, P.R.Briddon, M.J.Rayson et al., Phys. Rev. B, 72, 035214 (2005).

43. Y.G.Zhang, Z.Tang, X.G.Zhao et al., Appl. Phys. Lett., 105, 052107 (2014).

44. A.L.Pushkarchuk, S.A.Kuten, V.A.Pushkarchuk et al., in: Proc. XIV Int. Conf. on Quantum Optics and Quantum Information, Minsk, Belarus, 48 (2015). 